\title{
Fabrication and Properties of Composite Artificial Muscles Based on Nylon and a Shape Memory Alloy
}

\author{
Haibin Yin, Jia Zhou, Junfeng Li, and Vincent S. Joseph
}

\author{
(Submitted October 12, 2017; in revised form February 22, 2018; published online May 29, 2018)
}

\begin{abstract}
This paper focuses on the design, fabrication and investigation of the mechanical properties of new artificial muscles formed by twisting and annealing. The artificial muscles designed by twisting nylon have become a popular topic in the field of smart materials due to their high mechanical performance with a large deformation and power density. However, the complexity of the heating and cooling system required to control the nylon muscle is a disadvantage, so we have proposed a composite artificial muscle for providing a direct electricity-driven actuation by integrating nylon and a shape memory alloy (SMA). In this paper, the design and fabrication process of these composite artificial muscles are introduced before their mechanical properties, which include the deformation, stiffness, load and response, are investigated. The results show that these composite artificial muscles that integrate nylon and a SMA provide better mechanical properties and yield up to a $44.1 \%$ deformation and $3.43 \mathrm{~N}$ driving forces. The good performance and direct electro-thermal actuation make these composite muscles ideal for driving robots in a method similar to human muscles.
\end{abstract}

Keywords artificial muscle, nylon/SMA composites, shape memory alloy (SMA), twisting nylon

\section{Introduction}

The high cost of power and the redundant structures used in conventional rigid robots that have large inertia components restrict their applications in robots that require adaptability, security and efficiency (Ref 1-3). However, soft robots have the advantages of good adaptability, high security and efficiency due to the application of soft muscles with a high power density. Therefore, many researchers are attracted to studying the soft robots, where the research on artificial muscles is a major issue.

In the literature on artificial muscles, there are various types of actuation. Suzumori et al. designed a bending pneumatic rubber actuator to realize a soft-bodied manta swimming robot (Ref 4); Sasaki also studied the pneumatic soft actuator to drive the active support splint (Ref 5). Pneumatic muscles using air are common and cheap, but they result in a poor operation environment due to high levels of noise. Compared to pneumatic muscles, hydraulic muscles are expensive but have the advantages of low noise levels and good mechanical properties during actuation. Ku designed a hydraulic muscle to actuate underwater robots (Ref 6); Mori et al. also developed a large force-density robot hand using hydraulic muscles (Ref 7). Both the pneumatic and the hydraulic muscles adopt fluid mediums to adjust the inner pressure of a soft cavity and easily realize the actuation. However, they require the use of

Haibin Yin, Jia Zhou, and Junfeng Li, Key Laboratory of Hubei Province for Digital Manufacture, School of Mechanical and Electric Engineering, Wuhan University of Technology, Wuhan 430070, China; and Vincent S. Joseph, Engineering Product Development, Singapore University of Technology and Design, Changi 487372, Singapore. Contact e-mail: chinaliuyin@whut.edu.cn. additional pumps and control systems, so the whole actuators are complex systems with a low power density and efficiency.

Direct electricity-driven dielectric elastomeric actuators (DEA) (Ref 8) and electro-active polymer actuators (EAP) (Ref 9) are attractive because of their high power density and efficiency. Lu et al. developed a fiber-like DEA by using a high voltage to realize the high required electric fields (Ref 10), but the high voltage makes the muscle-like fibers difficult to work with safety. Due to the high voltage limits for the application of muscles in cases requiring low-cost and high-security actuators (Ref 11), the low-voltage-driven EAP actuator is studied to design high-security soft robots with a low cost, but the developed robots do not have a high load capacity (Ref 12).

Therefore, high-power-density, low-cost and high-strength fibrous artificial muscles are required. The SMA meets these demands in some applications, such as a soft composite structure SMA actuator (Ref 13), a SMA muscle (Ref 14) and a SMA mirror actuator (Ref 15); nevertheless, it has the shortcoming of having a small deformation of less than $7 \%$ to limit the range of its application (Ref 16). Alternatively, the artificial muscles from a twisting nylon line enlarge deformations, and the extreme twisting produces coiled nylon muscles that can contract by $49 \%$ (Ref 17). These nylon muscles were applied as actuated fingers (Ref 18) and coiled nylon muscles (Ref 19). However, these coiled nylon fibers are actuated by hot and cold fluid, such as air and water, within a glass tube, which is complex and restricts the application to the design of compact robots (Ref 20). Some researchers fabricated coiled nylon fibers by twisting nylon with a thin metal coating (Ref $21,22)$, through which a current is applied to generate heat and allow good control (Ref 23). The coiled nylon fiber is also wrapped by insulated copper wire for electro-thermal actuation (Ref 24). Although the electro-thermal actuation is a simple control system, the coiled nylon fibers with metal coating or copper wire wrapping have a low strength because the host material is nylon.

This paper proposes a new method of electro-thermal actuation by combining a high-strength SMA and large- 
deformation coiled nylon as fibrous composite artificial muscles. First, the fibrous muscles are designed, and the fabrication process is described. Then, four different methods and configurations of the composite muscles are introduced to obtain a deeper understanding of their fabrication process and properties. Further, a series of experiments are performed to investigate the fabrication processing and mechanical properties of the composite muscles. The final experimental results show that the proposed composite muscles perform well. The study of the mechanical and processing properties of the composite muscles will help us to design and manufacture suitable muscles in the developments of soft robots.

\section{Fabrications of Nylon/SMA Composite Muscles}

\subsection{Processing of Nylon/SMA Composite Muscle}

The manufacturing process and specimen model of the nylon/SMA composite muscles are presented in Fig. 1, where four artificial muscle specimens are fabricated with different processes. Twisted nylon line that can achieve contraction was discovered by Haines (Ref 17). As shown in the left side of Fig. 1, the experimental setup used to build twisted nylon is composed of a base, a motor, a motor driver, a weight and a power converter, where the motor is used to twist the nylon line, and the number of twisting turns $(N)$ can be controlled by the motor driver.

Haines' method could yield a coiled nylon $(\mathrm{CN})$ with a tight state, and the tight $\mathrm{CN}$ can be used in need of pre-stretching and a heating SMA that pass through its inner hole, as shown in the first row in Fig. 1. We call this composite muscle an innerradiation heating tight muscle (IHTM).

The $\mathrm{CN}$ is obtained throughout the auto-coiling process and requires a large number of twisting turns. While the nylon line is only twisted to the critical turns $\left(N_{\mathrm{c}}\right)$, which represents the number of twisting turns before the nylon line begins to coil automatically, the twisted nylon is manually coiled on an iron rod and fixed to clamps for annealing. A loose coiled muscle is obtained and is also heated by an inner-insert SMA wire; this composite muscle is called an inner-radiation heating loose muscle (IHLM).

Both the IHTM and the IHLM were actuated by the SMA, which is mainly used as a heater because the contraction of the coiled nylon dominates. The SMA can also produce thermalcontraction due to electro-thermal effects (Ref 18). Therefore, the coiled nylon and the SMA wire were combined to produce a composite functional muscle, which undergoes contraction resulting from the SMA and the coiled nylon.

This paper proposes a double-wire twisting process, as shown in the lower part of the first column in Fig. 1. The detailed processing procedure is depicted in Fig. 2, where the red line represents the nylon, and the yellow wire is the SMA. First, both ends of the nylon line and both ends of a longer SMA wire are tied together. Then, their top ends are fixed to the motor, and their bottom ends are attached to the weight. Second, the nylon and the SMA are twisted together as one composite precursor fiber, as shown in Fig. 2(b); the doublewire precursor fiber is twisted to the critical turns and begins to coil, as shown in Fig. 2(c). Finally, a coiled muscle is obtained and called a combined contraction tight muscle (CCTM), where the SMA wires not only heat the coiled nylon to actuate it but also contract with the coiled nylon.

Similarly, when the above-mentioned double-wire precursor fiber is twisted to its critical turns, the twisted precursor fiber is manually coiled on an iron rod and fixed to clamps for annealing. Then, a double-wire loose muscle is obtained and called a combined contraction loose muscle (CCLM).

\subsection{Specimens of Composite Muscles}

We have introduced four types of fabrication processes for composite muscles. In addition to the processes, the fabrication of composite muscles requires the specification of the composition and dimensions of the materials, the weight and the annealing temperature.



Fig. 1 Manufacturing processes and specimen model of composite muscles 


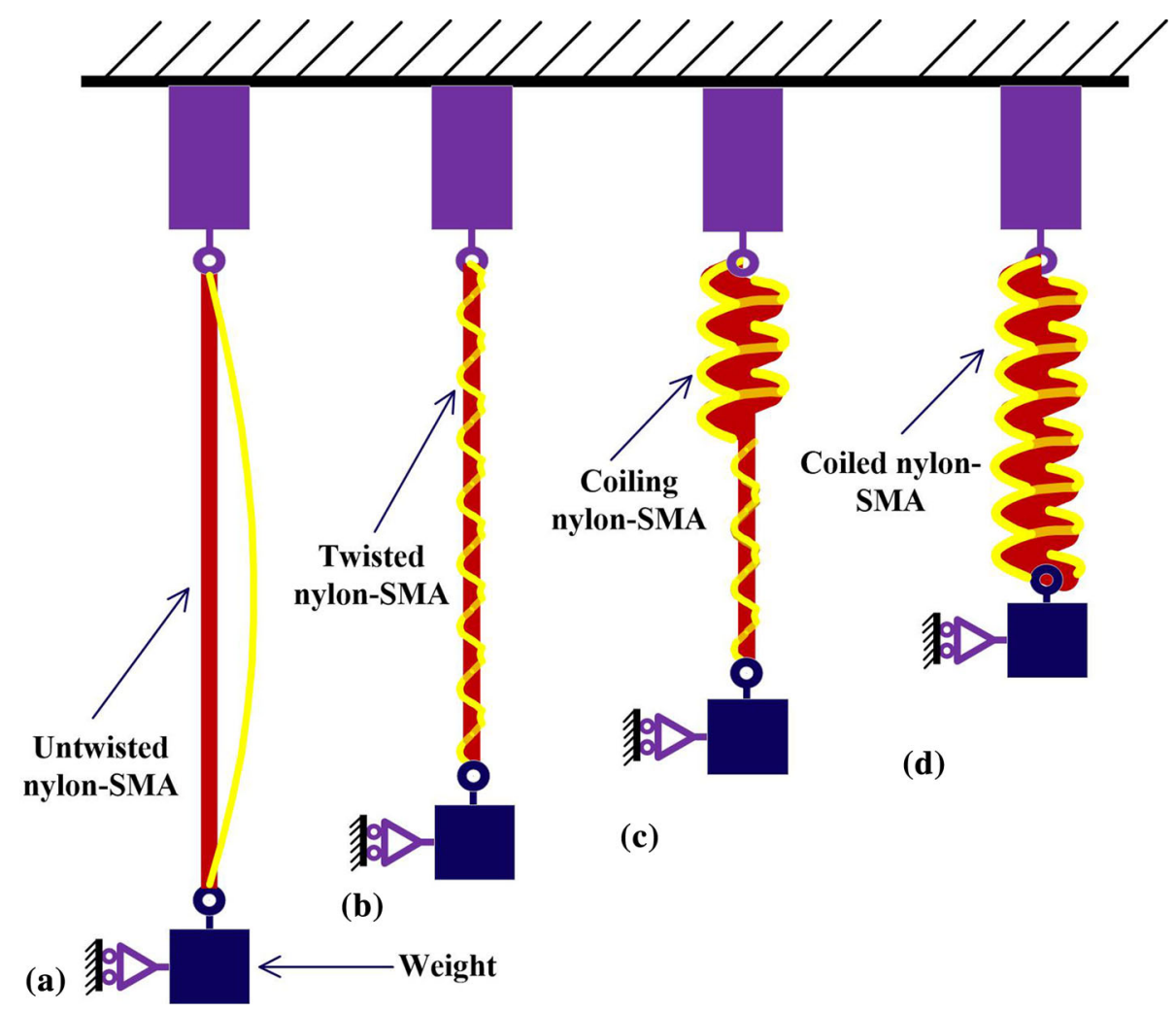

Fig. 2 Scheme of the auto-coiling process for double-wire composite muscles: (a) The two ends of both the nylon line and the SMA wire are tied together and have their top ends fixed to a motor and their bottom ends attached to a weight. (b) The nylon line and the SMA wire are twisted together as one composite precursor fiber. (c) As the precursor fiber is twisted to the critical turns, it will begin to coil. (d) Finally, a coiled composite muscle is obtained

In the fabrication, the raw material was chosen as Nylon 6.6 due to its high melting point, and a nickel titanium (NiTi) SMA was chosen as the heating component due to its large strain (up to $7 \%$ ).

In the fabrication of an IHTM, the top end of a nylon line with a diameter of $0.5 \mathrm{~mm}$ is attached to the servomotor, and its bottom end is fixed to a 350-g weight to obtain the coiled nylon. A SMA wire with a diameter of $0.3 \mathrm{~mm}$ is inserted in the inner hole of the coiled nylon to form the composite muscle, as shown in Fig. 3(a), where the SMA wire can produce much electro-thermal radiation but undergoes a smaller contraction than the pre-stretching coiled nylon.

However, the main drawback of an IHTM is that it undergoes little deformation because the tightly coiled nylon requires pre-stretching generated by a payload, which also prevents the deformation of the IHTM (Ref 15). Therefore, an IHLM is fabricated by twisting a nylon line with a diameter of $0.5 \mathrm{~mm}$ to the critical turns and manually coiling it on an iron rod with a diameter of $1 \mathrm{~mm}$ for annealing. The nylon coiled on the iron rod is fixed to the clamps and then heated to $95{ }^{\circ} \mathrm{C}$ for $1 \mathrm{~h}$ in a high-precision heat treatment furnace (DHG-9030A), and then the shape of the coiled nylon can be fixed to the loose state, as shown in Fig. 3(b). Similarly, the IHLM is electrothermally actuated by the inner-insert SMA wire with a diameter of $0.3 \mathrm{~mm}$.

To compare the properties of artificial muscles with both inner-radiation heating muscles (IHTM and IHLM), both of the combined contraction muscle (CCTM and CCLM) specimens are fabricated according to the fabrication process shown in Fig. 1.
In the fabrication process of the CCTM, the nylon line with a diameter of $0.5 \mathrm{~mm}$ and the SMA wire with a diameter of $0.15 \mathrm{~mm}$ are attached together at both ends, and then their top ends are fixed to the twisting motor, and their bottom ends are attached to the $350 \mathrm{-g}$ weight to form a double-wire precursor fiber. The relative length between the nylon line and the SMA wire is the key problem in the fabrication. If the length of the SMA wire is no more than the length of the nylon line, the SMA will break during the twisting due to its low stretch ability. If the length of the SMA wire is much larger than the length of the nylon line, they will be combined in a state of disorder. Only if the length of SMA wire is 1.014 times that of the nylon line will the SMA wire be tightly coiled on the nylon line without breaking and finally form a fine CCTM, as shown in Fig. 3(c).

Similarly, a CCTM also has the same characteristics as an IHTM, which requires a pre-stretching payload to obtain the limited deformation. Therefore, the CCLM was fabricated by twisting the above-mentioned double-wire precursor to its critical turns and then manually coiling it on the iron rod for annealing at $95{ }^{\circ} \mathrm{C}$ for $1 \mathrm{~h}$. The CCLM specimen is obtained as shown in Fig. 3(d).

\section{Processing Properties of Composite Muscles}

The four specimens of composite muscles are fabricated with specified parameters for the materials, the weight and the annealing temperature, while different parameters will signif- 
(a) IHTM

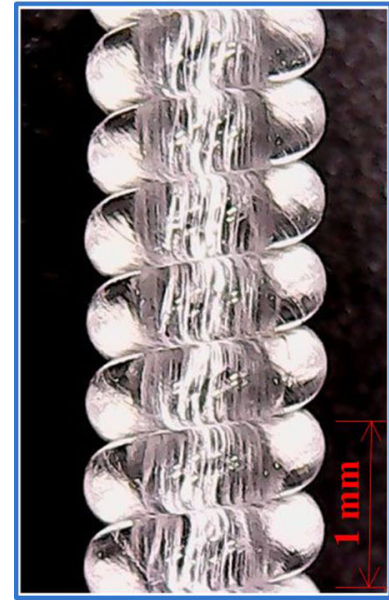

(b) IHLM

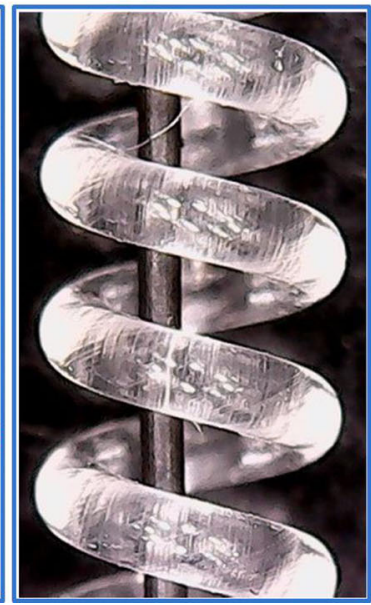

(c) ССТМ

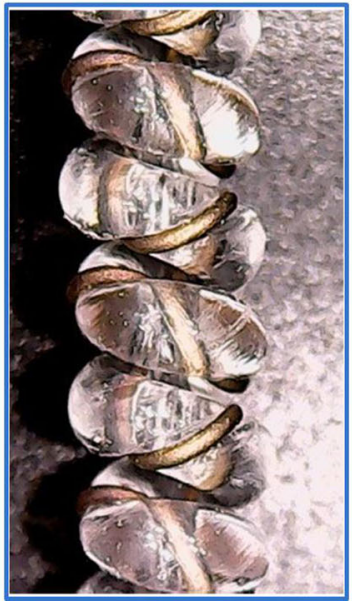

(d) CCLM

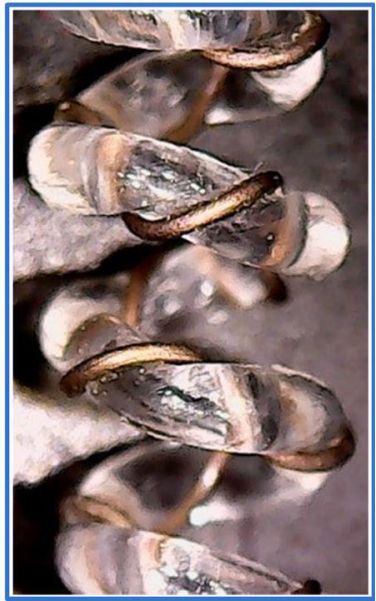

Fig. 3 Optical images of four specimens of composite muscles: (a) IHTM fabricated by twisting 0.5 mm nylon line and being heated by a $0.3 \mathrm{~mm}$ SMA wire, (b) IHLM fabricated by twisting $0.5 \mathrm{~mm}$ nylon line to the critical turns and coiling it on the iron rod with a diameter of $1 \mathrm{~mm}$ for annealing and being heated by a $0.3 \mathrm{~mm}$ SMA wire, (c) CCTM fabricated by twisting $0.5 \mathrm{~mm}$ nylon line and $0.15 \mathrm{~mm}$ SMA wire, (d) CCLM fabricated by twisting $0.5 \mathrm{~mm}$ nylon line and $0.15 \mathrm{~mm}$ SMA wire to the critical turns and coiling it on the iron rod with a diameter of $1 \mathrm{~mm}$ for annealing

icantly affect the processing properties. In this section, a series of experiments on the processing properties related to these parameters, such as dimensions of materials, weight and annealing temperature, are investigated.

\subsection{Spring Index}

Primarily, we need to investigate the main dimensions of composite muscles affected by the weights and the critical turns.

The spring index $C$ of the coiled nylon, which is a main dimension for describing the composite muscles and affected by the weights, is defined as follows:

$C=\frac{D}{d}$

where $D$ is the diameter of the coiled nylon and $d$ is the diameter of the nylon line.

Three kinds of nylon lines with the same length $(650 \mathrm{~mm})$ and different diameters $(0.5,0.4$ and $0.3 \mathrm{~mm})$ are adopted in this test. The spring indexes are measured after the coiled nylons with good quality form under the action of the weights within a certain range. As shown in Fig. 4, the spring indexes of these coiled nylons with good quality decrease with the increase in the weight.

\subsection{Weights and Critical Turns}

However, the good processing quality of composite muscles is also related to the weights attached at the bottom ends of twisted nylon lines and their critical turns. In these tests, three specimens are fabricated using the nylon lines with the same length of $650 \mathrm{~mm}$ and diameters of $0.5,0.4$ or $0.3 \mathrm{~mm}$. Figure 5 shows the measured results of the critical turns $\left(N_{\mathrm{c}}\right)$ for the various weights. The overall trends of these measured results are that the critical turns of the nylon lines increase with the increase in the weight. However, the three kinds of twisted nylon lines would produce flaws if the weights are lower than 200, 130 and $40 \mathrm{~g}$. Moreover, the three kinds of twisted nylon

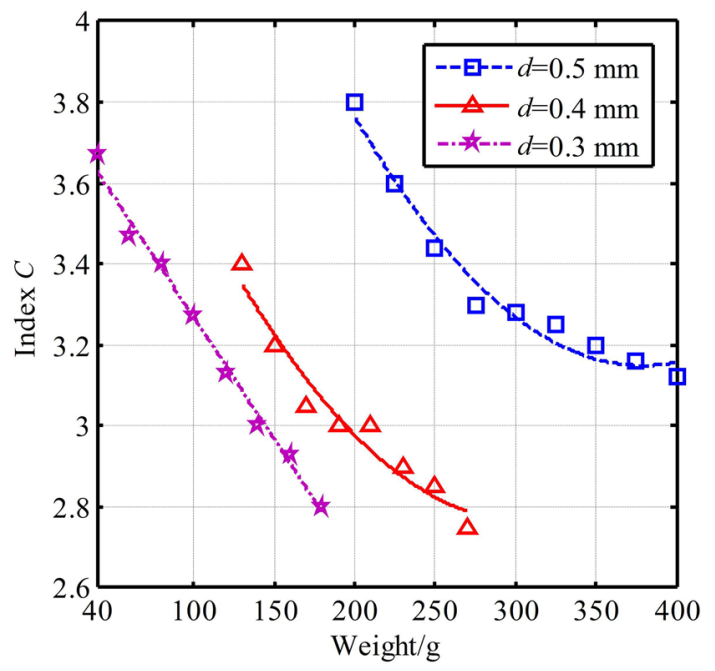

Fig. 4 Relationship between the spring indexes and weights attached at the bottom ends of the nylon lines with different diameters

lines would break before beginning to coil if the weights are larger than 400, 270 and $180 \mathrm{~g}$. Therefore, the weight and the critical turns of the nylon lines, which are closely related to the good processing quality of composite muscles, must be reasonably considered in fabrication.

\subsection{Annealing Temperature}

The annealing process is used to eliminate the internal stress of coiled nylon and make the loose muscle by fixing its shape in furnace. However, the loose muscle will retract after it is removed from the fixed clamps at the end of the annealing process. The retraction percentage of the loose muscle is related to the annealing temperature, so the relationship between the retraction percentage and the annealing temperature should be investigated. The loose muscles are fabricated under a given weight $(350 \mathrm{~g})$, so the required critical turns are known 


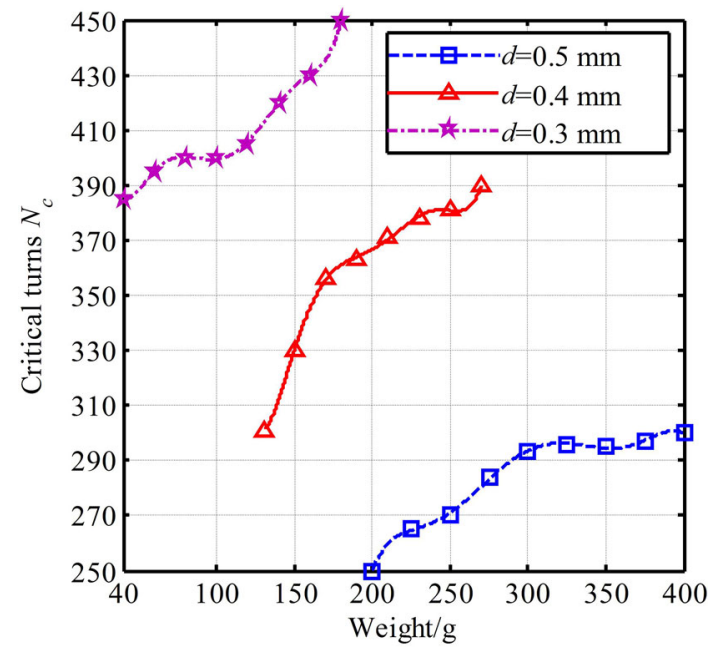

Fig. 5 Relationship between the critical turns and the weights attached at the bottom ends of the nylon lines with different diameters

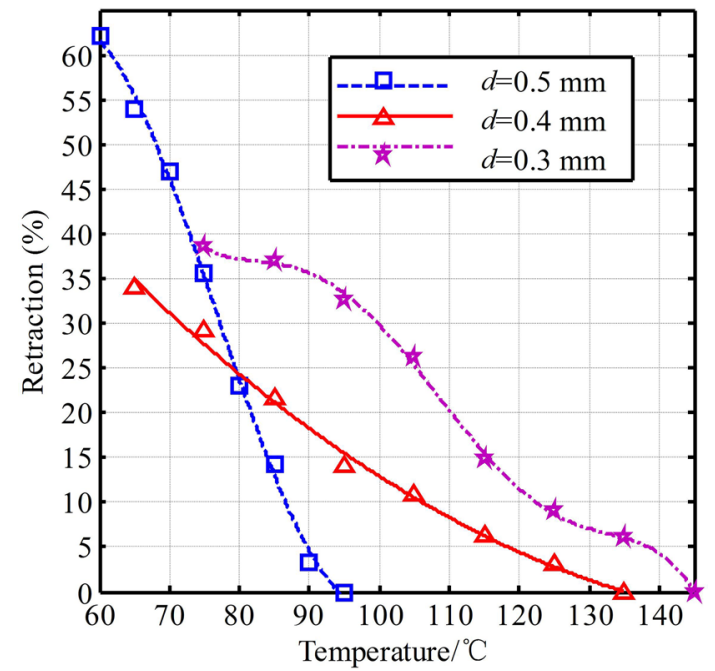

Fig. 6 Relationship between the retraction percentage and the annealing temperature of loose muscles with different diameters

according to the results in Fig. 4. In this test, the annealing temperature is set to different values for $1 \mathrm{~h}$, and then these loose muscles are obtained and placed freely at room temperature for $5 \mathrm{~h}$. Their retraction percentages are measured as shown in Fig. 6, where the retraction percentage decreases with the annealing temperature. Moreover, the retraction percentage almost disappears when the annealing temperatures are set up to 95,135 and $145^{\circ} \mathrm{C}$ for the nylon lines with diameters of 0.5 , 0.4 and $0.3 \mathrm{~mm}$. These results can be used to determine the annealing temperature for eliminating the nylon internal stress and fixing the shape of loose artificial muscles.

\subsection{Inserting Turns}

In the aforementioned tests, these loose muscles are fabricated by twisting the nylon line to its critical turns and then manually coiling it on the iron rod to fix the shape. If the twisting turns of the nylon line before it is manually coiled on the iron rod, which is named the inserting turns $\left(N_{\mathrm{i}}\right)$, are fewer than its critical turns $\left(N_{\mathrm{c}}\right)$, what is the impact on the mechanical

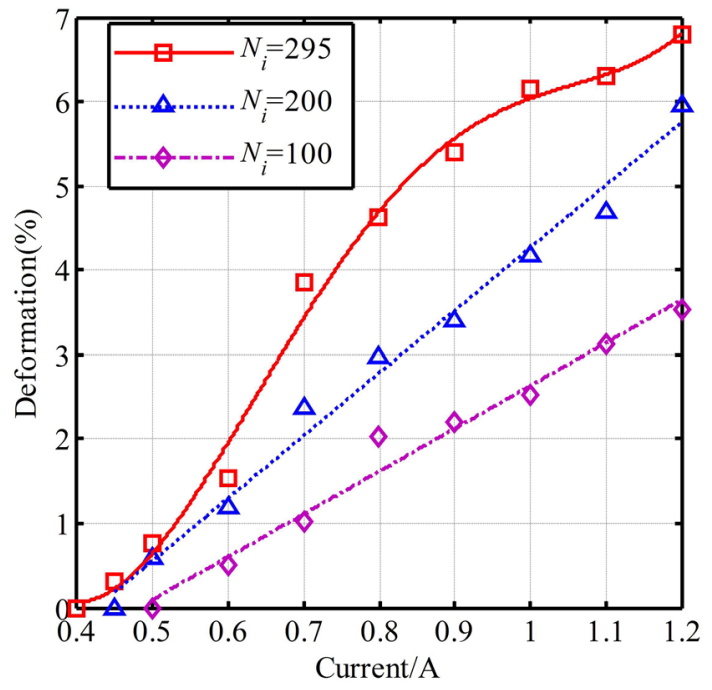

Fig. 7 Relationship between deformation and the current of the loose muscles with different inserting turns

properties of the corresponding loose muscle? To explore this problem, three types of specimens are fabricated by twisting the nylon line with a diameter of $0.5 \mathrm{~mm}$ to 100,200 and 295 turns and then manually coiling it on the iron rod to fix the shape. After obtaining these loose muscles (IHLM), their deformations are measured under the action of a heating current; the results are presented in Fig. 7. These results depict that the deformation of the IHLM increases with the inserting turns and that the IHLM with 295 inserting turns, which is also the critical turns according to the results in Fig. 4, has the maximum deformation capacity. As a result, the deformation of the loose muscles inserted critical turns will be introduced in the upcoming mechanical properties section.

\section{Mechanical Properties of Composite Muscles}

In conclusion, IHTM and CCTM are auto-coiling tight muscles, while IHLM and CCLM are manual-coiling loose muscles. The latter require inserting turns before they are manually coiled on the iron rod, and the loose muscles inserted critical turns have the maximum deformation capacity. Therefore, the loose muscles specimens are fabricated by inserting the critical turns to test their mechanical properties. In this section, a series of tests on the mechanical properties, such as deformation, stiffness, mechanical characteristics and dynamic response, of the composite muscles, are conducted.

\subsection{Deformation}

Deformation is defined as the shrinkage ratio $(\varepsilon)$ of the composite muscles. Figure 8 shows the experimental setup and the test scheme. According to the test scheme and the definition, the deformation of a composite muscle is described as follows:

$\varepsilon=\frac{\Delta L}{L}$

where $L$ is initial length and $\Delta L$ is the shrinkage length of the composite muscle. 

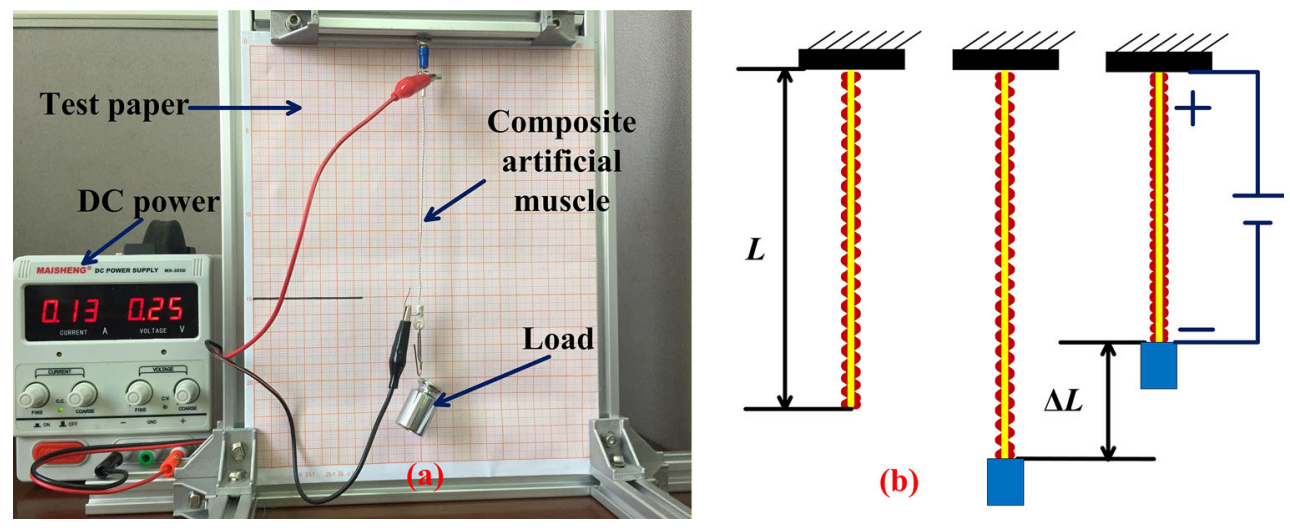

Fig. 8 (a) Experimental setup and (b) test scheme for deformation of composite muscles
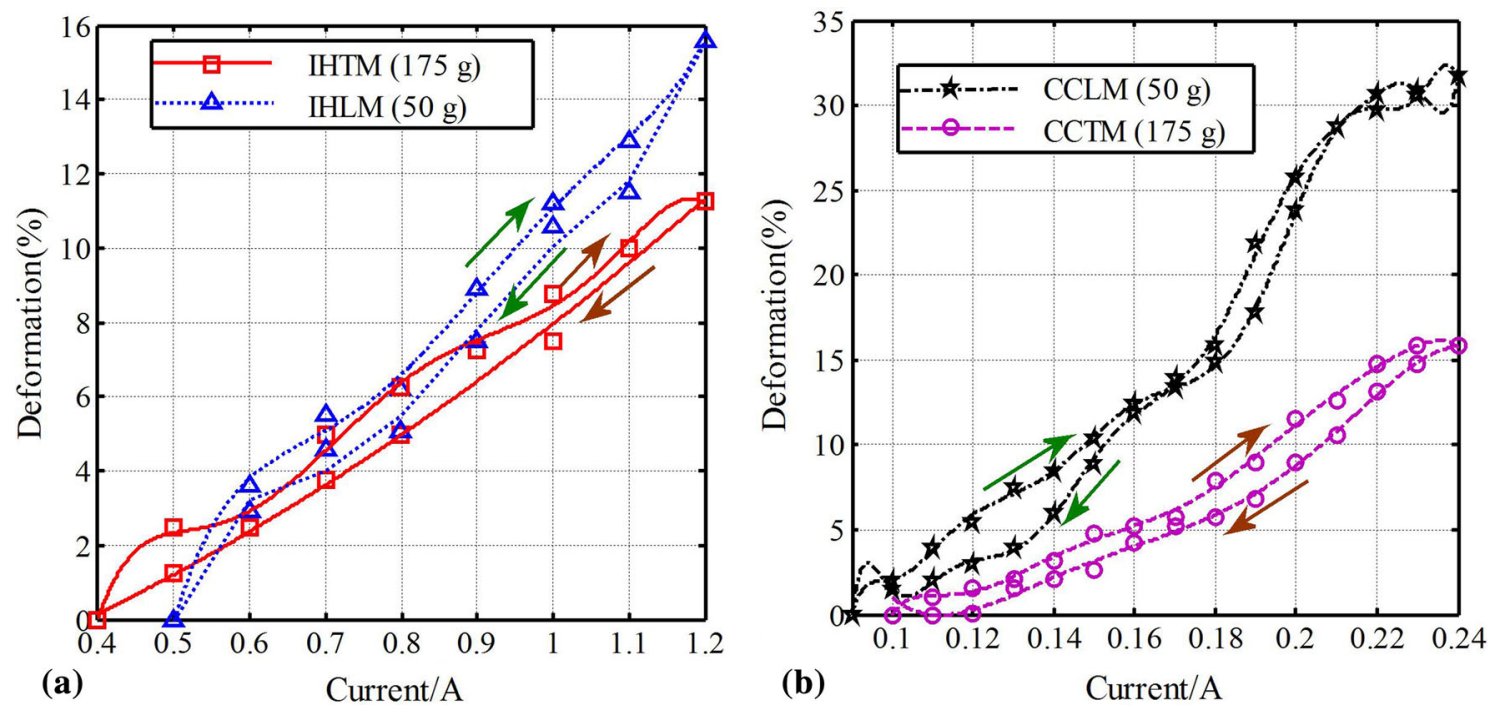

Fig. 9 Deformation of (a) IHTM and IHLM, (b) CCTM and CCLM under the action of currents

These deformations of IHTM, IHLM, CCTM and CCLM are measured by loading $175,50,175$ and $50 \mathrm{~g}$, respectively. The tests on the IHTM and IHLM are conducted by charging the inner-insert $0.3 \mathrm{~mm}$ SMA wire with currents ranging from $0.4 \mathrm{~A}$ (initial input current) to $1.2 \mathrm{~A}$, while the tests on the CCTM and CCLM are conducted by charging the $0.15 \mathrm{~mm}$ SMA wire with currents ranging from 0.08 to $0.24 \mathrm{~A}$. These measured data are analyzed, and the results are depicted in Fig. 9, where each deformation is shown in a closed line, with the heating and cooling routes marked with arrows corresponding to the increase and decrease in the current.

As shown in Fig. 9, the maximum deformations of the IHTM, IHLM, CCTM and CCLM are 11.25, 15.53, 15.79 and $31.68 \%$ corresponding to given loads, respectively. We can summarize that the composite muscle has a larger deformation capacity if the load is lighter. The load at the bottom of the composite muscles seems to decrease their deformation capacity, so we need to further investigate their mechanical characteristics.

\subsection{Mechanical Characteristics}

To discuss the relationship between the load and the deformation capacity of composite muscles, the maximum deformations of the IHTM and IHLM related to the loads are measured within 1.2 A because the larger current will result in breaking of the nylon lines; additionally, the maximum deformations of the CCTM and CCLM related to the loads are measured within $0.24 \mathrm{~A}$ because the larger current will melt the nylon lines. The measured results between the maximum deformation and the loads are shown in Fig. 10.

The maximum deformations of loose muscles (IHLM and CCLM) decrease with the increase in the loads, while the maximum deformations of tight muscles (IHTM and CCTM) first increase with loads due to the requirement of pre-stretching and then decrease with the increase in the loads. As shown in Fig. 10, the critical values of the pre-stretching weights are 110 and $150 \mathrm{~g}$. The maximum deformations of the IHTM, IHLM, CCTM and CCLM are 25.6, 37.5, 20.7 and 44.1\%, and their maximum loads $\left(G_{\max }\right)$ or driving forces are 190, 80, 350 and $150 \mathrm{~g}$, respectively. As a result, the composite muscle with maximum deformations does not have to obtain the maximum driving force.

\subsection{Stiffness}

In this section, the stiffness $K$ of the composite muscles is defined as the pulling force to produce a unit stretching 


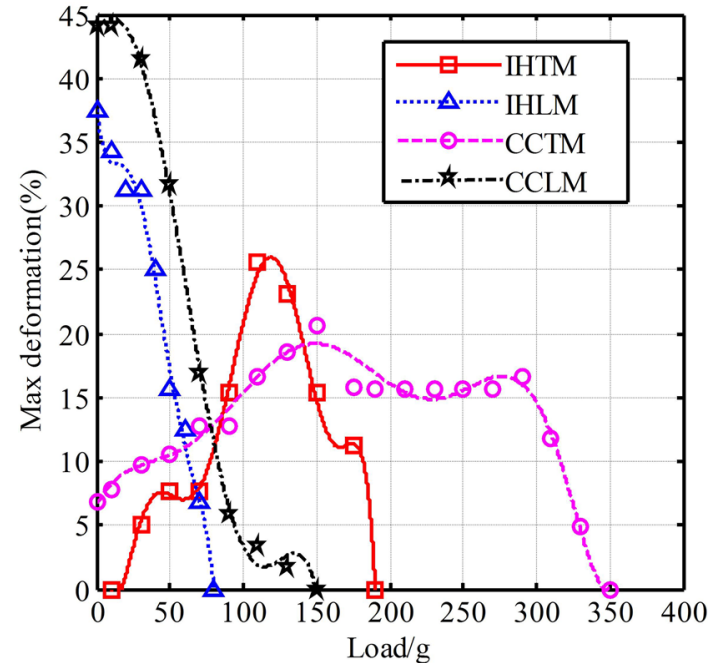

Fig. 10 Relationship between the maximum deformation of composite muscles and the loads

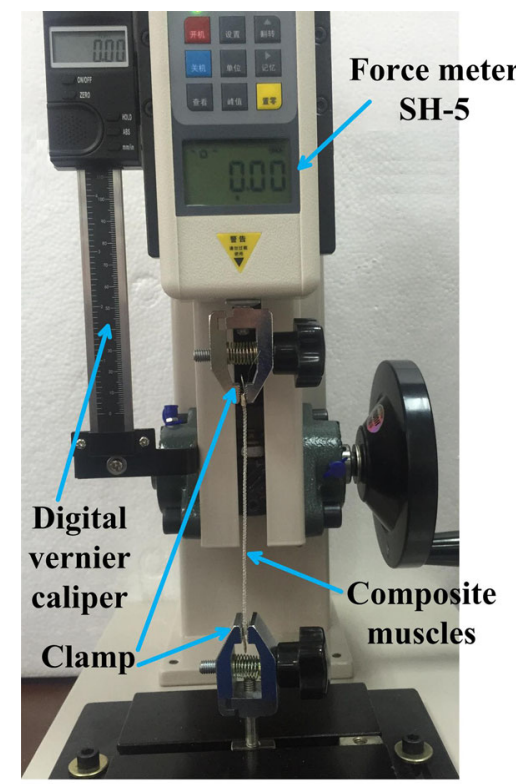

(a)

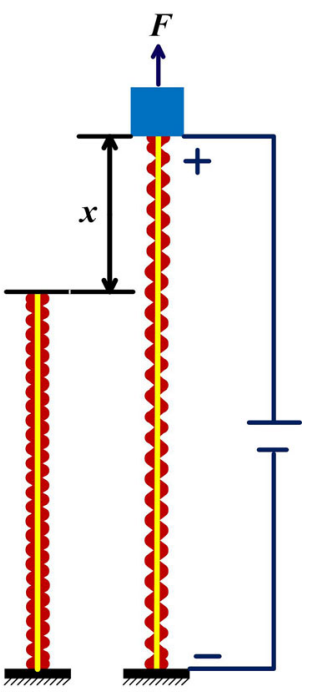

(b)

Fig. 11 (a) Experimental setup and (b) test scheme for the stiffness of composite muscles

deformation. Figure 11 depicts the experimental setup and the test scheme to measure the stiffness. According to the definition and the test scheme, the stiffness of the composite muscles is represented by:

$K=\frac{F}{x}$

where $F$ is the pulling force and $x$ is the stretching deformation.

The stretching deformation $x$ is measured by a digital Vernier caliper, and the pulling force $F$ is measured by a force meter (SH-5) under different currents.

Figure 12 depicts the stiffness results of composite muscles. All stiffness values of the IHTM, IHLM, CCTM and CCLM decrease with the increase in the current, and their maximum values are $0.176,0.085,0.15$ and $0.03 \mathrm{~N} / \mathrm{mm}$, respectively. These stiffness values of loose muscles (IHLM and CCLM) have better linearity than those of tight muscles under the action of currents, while the stiffness values of tight muscles (IHTM and CCTM) are larger than those of loose muscles in the whole range of the working currents.

\subsection{Dynamic Response}

The previous properties are measured for static cases, disregarding the dynamic factors that are required in the application of dynamic control. In the section, the dynamic responses of composite muscles are evaluated using the experimental setup and test scheme, as shown in Fig. 13. The experimental setup is composed of the DC power supply, a force sensor and the ARDUINO controller, where the heating current is controlled using a switch to keep the heating synchronized to the sampling.

First, the positions of the force sensors are adjusted to obtain the same initial value $(0.1 \mathrm{~N})$ of tensions for the four kinds of composite muscles, and the initial value is set to 0 . Then, the tensions of the four kinds of composite muscles rise under the action of the heating current and are measured. Due to the different heating modes, the tensions of IHTM and IHLM are tested under a 1.2-A current, and the tensions of CCTM and CCLM are tested under a 0.24-A current. The tensions are measured as incremental values, which show the transition functions $(f)$ tracking step objectives, as shown in Fig. 14.

In these tests, the dynamic response is described as a time constant, which reflects the response speed in tracking the objective tensions of the composite muscles. According to the model of the transition process, the time constant is represented by:

$\tau_{m}=\frac{f(\infty)}{f^{\prime}(0)}$

where $f(\infty)$ is the objective value of the tension, and $f^{\prime}(0)$ denotes the value of the derivatives of the transition function at the initial time.

Based on the experiment results, these time constants of the IHTM, IHLM, CCTM and CCLM are calculated as 33.6, 13.5, 10.7 and $22.4 \mathrm{~s}$, corresponding to the objective tensions of 0.4 , $0.2,1.28$ and $0.55 \mathrm{~N}$, respectively. As a result, the CCTM muscle fiber appears to exhibit a good dynamic performance under the action of a large payload.

\subsection{Results and Discussion}

There are four specimens of composite muscles, which are classified into two groups according to the heating mode. The mechanical properties of the composite muscles are summarized in Table 1. In each group, the loose muscles have a larger deformation capacity, while the tight muscles have a larger driving capacity and stiffness. The CCLM muscle fiber can yield up to $44.1 \%$ deformation, which is larger than the $7 \%$ deformation resulting from the SMA (Ref 16), and the CCTM muscle can drive up to a 3.43-N load, which exceeds these values in similar works, such as the $2-\mathrm{N}$ driving force based on the coiled nylon muscle made by Wu (Ref 18) and the 1.2-N driving force resulting from the silver-painted nylon muscle designed by Mirvakili (Ref 21).

To evaluate the work capacity of composite muscles, a new parameter is defined as $P=\varepsilon_{\max } G_{\max }$, which reflects the 

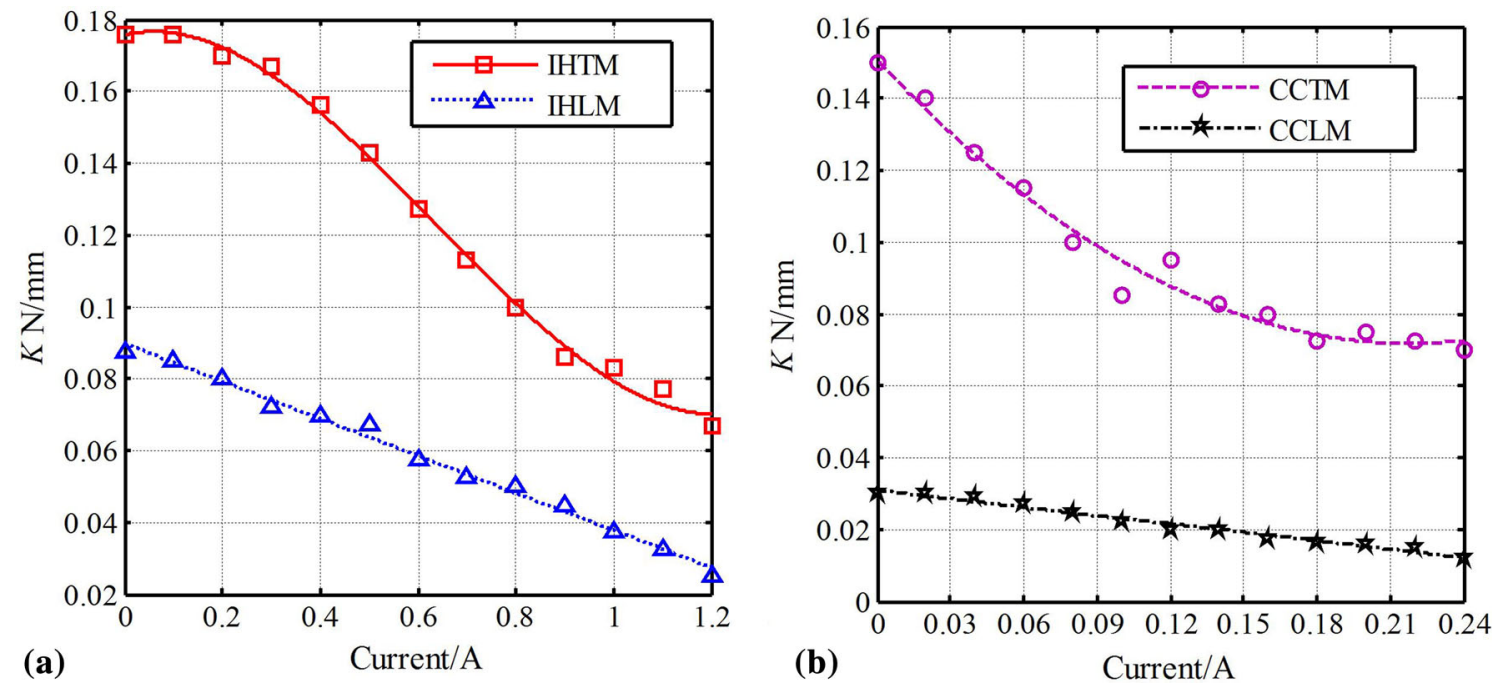

Fig. 12 Stiffness results of (a) IHTM and IHLM, (b) CCTM and CCLM under the action of currents

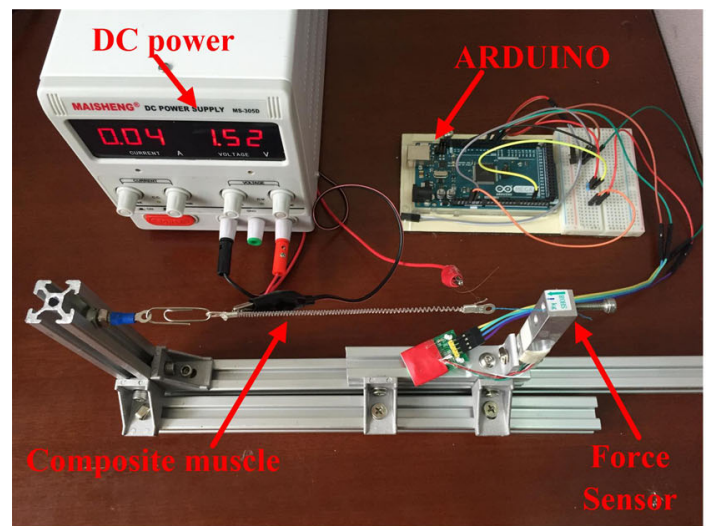

(a)

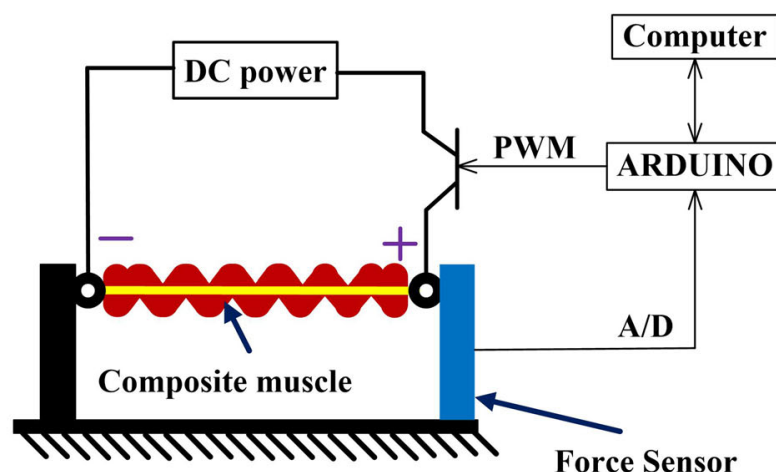

(b)

Fig. 13 (a) Experimental setup and (b) test scheme for dynamic response of composite muscles

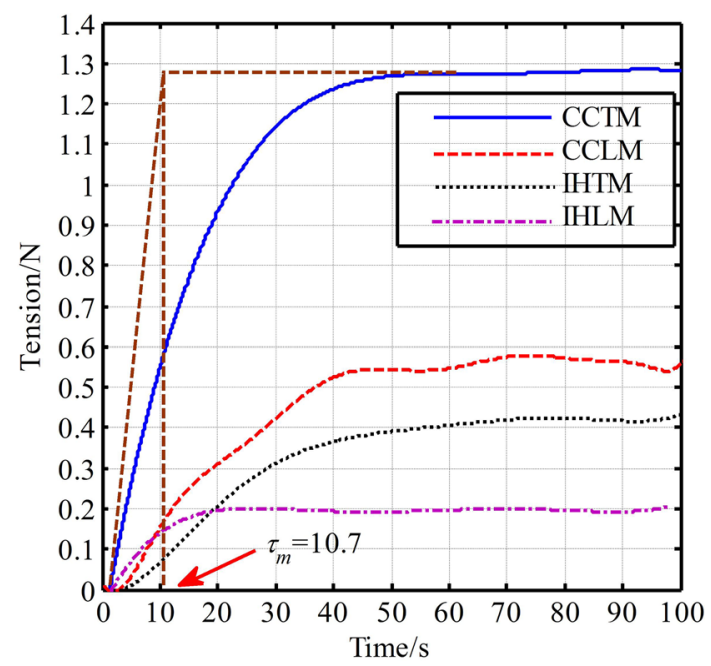

Fig. 14 Transition functions of the tension of composite muscles and the time constant maximum output power of a unit length muscle and is named the linear power density. The values of the linear power density of the composite muscles (IHTM, IHLM, CCTM and CCLM) are $4.86,3.00,7.25$ and $6.62 \mathrm{~W} / \mathrm{m}$, respectively. As a result, the CCTM has the maximum linear power density and response speed of the four types of specimens.

\section{Conclusions}

This paper has proposed a new electro-thermal actuator by combining a high-strength SMA with a large-deformation coiled nylon as fibrous composite muscles. Four specimens, called IHTM, IHLM, CCTM and CCLM, were designed and fabricated by twisting and annealing. Then, the processing and mechanical properties were measured and analyzed.

Based on the analysis of the processing properties, we deduce that the fabrications of composite muscles are dependent on their parameters, such as the dimensions of the materials, the load, the annealing temperature and the twisting 
Table 1 Mechanical properties of composite muscles

\begin{tabular}{lcccc}
\hline Specimens name & Deformation $\boldsymbol{\varepsilon}_{\mathbf{m a x}}, \boldsymbol{\%}$ & Load $\boldsymbol{G}_{\mathbf{m a x}}, \mathbf{k g}$ & Time constant $\boldsymbol{\tau}_{\boldsymbol{m}}, \mathbf{s}$ & Power density $\boldsymbol{P}, \mathbf{W} / \mathbf{m}$ \\
\hline IHTM & 25.6 & 0.19 & 33.6 & 4.86 \\
IHLM & 37.5 & 0.08 & 13.5 & 3.00 \\
CCTM & 20.7 & 0.35 & 10.7 & 7.25 \\
CCLM & 44.1 & 0.15 & 22.4 & 6.62 \\
\hline
\end{tabular}

turns. From the analysis of the mechanical properties, we summarize that the composite muscles could yield up to a $44.1 \%$ deformation and drive up to $3.43-\mathrm{N}$ loads; moreover, the composite muscle CCTM could provide up to a $6.77 \mathrm{~W} / \mathrm{m}$ power density and a fast dynamic response.

The experimental data showed that the proposed composite muscles have good properties and the potential to increase the performance of soft robots. One of the challenges for further research is an exploration of more practical applications, such as fingers, robotic actuation and wearable devices.

\section{Acknowledgments}

This work was supported by the National Natural Science Fundament of China (NSFC) under Grant [No. 51575409].

\section{References}

1. W. Wang, H. Rodrigue, H.I. Kim et al., Soft Composite Hinge Actuator and Application to Compliant Robotic Gripper, Compos. Part B Eng., 2016, 98, p 397-405

2. H. Lin, M.J. Clifford, P.M. Taylor et al., 3D Mathematical Modelling for Robotic Pick Up of Textile Composites, Compos. Part B Eng., 2009, 40(8), p 705-713

3. H.J. Kim, S.H. Song, and S.H. Ahn, A Turtle-Like Swimming Robot Using a Smart Soft Composite (SSC) Structure, Smart Mater. Struct., 2012, 22(1), p 014007

4. K. Suzumori, S. Endo, T. Kanda et al., A Bending Pneumatic Rubber Actuator Realizing Soft-Bodied Manta Swimming Robot, in 2007 IEEE International Conference on Robotics and Automation (IEEE, 2007), pp. $4975-4980$

5. D. Sasaki, T. Noritsugu, and M. Takaiwa, Development of Active Support Splint Driven by Pneumatic Soft Actuator (ASSIST), in 2005 IEEE International Conference on Robotics and Automation (IEEE, 2005), pp. 520-525

6. K.K.K. Ku, R. Bradbeer, and K. Lam, Exploration for Novel Uses of Air Muscles as Hydraulic Muscles for Underwater Actuator, in Oceans IEEE (2008), pp. 1-6

7. M. Mori, K. Suzumori, S. Wakimoto et al., Development of Power Robot Hand with Shape Adaptability Using Hydraulic McKibben Muscles, in 2010 IEEE International Conference on Robotics and Automation (IEEE, 2010), pp. 1162-1168
8. G. Rizzello, D. Naso, A. York et al., Closed Loop Control of Dielectric Elastomer Actuators Based on Self-Sensing Displacement Feedback, Smart Mater. Struct., 2016, 25(3), p 035034

9. Y. Barcohen, Electroactive Polymers: Current Capabilities and Challenges, Proc. SPIE, 2002, 4695, p 4692-4695

10. T. Lu, J. Huang, C. Jordi et al., Dielectric Elastomer Actuators Under Equal-Biaxial Forces, Uniaxial Forces, Uniaxial Constraint of Stiff Fibers, Soft Matter, 2012, 8(22), p 6167-6173

11. D.F. Hanson, EAP Artificial Muscle Actuators for Bio-inspired Intelligent Social Robotics (Conference Presentation), Proc. SPIE, 2017, 10163, p 1016302

12. S. Yun, J. Kim, and C. Song, Performance of Electro-active Paper Actuators with Thickness Variation, Sens. Actuators A, 2007, 133(1), p 225-230

13. S.H. Song, H. Lee, J.G. Lee et al., Design and Analysis of a Smart Soft Composite Structure for Various Modes of Actuation, Compos. Part $B$ Eng., 2016, 95, p 155-165

14. M.M. Ghomshei, N. Tabandeh, A. Ghazavi et al., A Three-Dimensional Shape Memory Alloy/Elastomer Actuator, Compos. Part B Eng., 2001, 32(5), p 441-449

15. E. Williams and M.H. Elahinia, An Automotive SMA Mirror Actuator: Modeling, Design, and Experimental Evaluation, J. Intell. Mater. Syst. Struct., 2008, 19(12), p 1425-1434

16. H.I. Kim, M.W. Han, S.H. Song et al., Soft Morphing Hand Driven by SMA Tendon Wire, Compos. Part B Eng., 2016, 105, p 138-148

17. C.S. Haines, M.D. Lima, N. Li, G.M. Spinks, J. Foroughi, J.D.W. Madden, and R.H. Baughman, Artificial Muscles from Fishing Line and Sewing Thread, Science, 2014, 343(6173), p 868-872

18. L. Wu, M.J. de Andrade, R.S. Rome et al., Nylon-Muscle-Actuated Robotic Finger, Proc. SPIE, 2015, 9431, p 943101

19. C. Antonello, M. Giacomo, V. Rocco, and F. Marco, Experimental Characterization of Thermally-Activated Artificial Muscles Based on Coiled Nylon Fishing Lines, AIP Adv., 2015, 5, p 1-11

20. J.D.W. Madden and S. Kianzad, Twisted Lines: Artificial Muscle and Advanced Instruments can be Formed from Nylon Threads and Fabric, IEEE Pulse, 2015, 6(1), p 32-35

21. S.M. Mirvakili, A.R. Ravandi, I.W. Hunter et al., Simple and Strong: Twisted Silver Painted Nylon Artificial Muscle Actuated by JOULE HEATING, Proc. SPIE, 2014, 9056, p 905601

22. L. Wu, M.J. de Andrade, L.K. Saharan et al., Compact and Low-Cost Humanoid Hand Powered by Nylon Artificial Muscles, Bioinspir. Biomim., 2017, 12(2), p 026004

23. C.S. Haines, N. Li, G.M. Spinks et al., New Twist on Artificial Muscles, Proc. Natl. Acad. Sci., 2016, 113, p 11709-11716

24. M.C. Yip and G. Niemeyer, High-Performance Robotic Muscles from Conductive Nylon Sewing Thread, in 2015 IEEE International Conference on Robotics and Automation (ICRA) (IEEE, 2015), pp. 2313-2318 